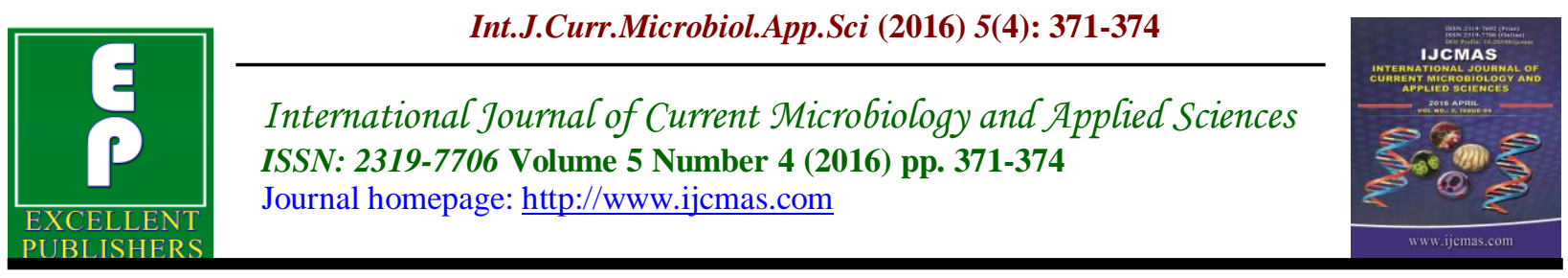

Original Research Article

http://dx.doi.org/10.20546/ijcmas.2016.504.044

\title{
Microbiological Corroboration for Diagnosis of VAP (Ventilator Associated Pneumonia) by Quantitative Culture of Endotracheal Aspirate (QEA) in ICU Patients in a Tertiary Hospital, South India
}

\author{
V. Jayashree*, Karthika Jayakumar and S. Parvathi \\ Department of Microbiology, Shri Sathya Sai Medical College \& Research Institute, \\ Kanchipuram, Tamil Nadu, India \\ Department of Microbiology, PSG Institute of Medical Sciences \& Research, Coimbatore, India \\ *Corresponding author
}

\section{A B S T R A C T}

Keywords

VAP, ICU,

Endotracheal aspirate,

Quantitative

culture,

CPIS score.

\section{Article Info}

Accepted:

15 March 2016

Available Online:

10 April 2016
Patients in the intensive care unit (ICU) are at risk, not only due to their critical illness but also from secondary processes such as nosocomial infections. Pneumonia is known to be the most common nosocomial infection among patients in ICUs. Rates of pneumonia are considerably higher among hospitalized patients, and the risk of developing pneumonia is 3-10 folds higher in ventilated patients. In critical care settings, within hours of endotracheal intubation, the upper respiratory tract is colonized by potential pulmonary pathogens. Thus, if an organism is cultured or seen on Gram's stain, one does not know, if it is the cause for pneumonia or simply colonization. Ventilator-associated pneumonia (VAP) increases the crude mortality rate by 2-10 times, and the hospital costs by increasing the length of stay and the need for more expensive antibiotics.

\section{Introduction}

Ventilator-associated pneumonia (VAP) is defined as pneumonia occurring more than 48 hours after patients have been mechanically ventilated by endotracheal intubation ${ }^{1}$. Diagnosing VAP requires a high clinical suspicion combined with bed-side examination, radiography and microbiological analysis of respiratory secretions. VAP is usually suspected when the patient develops a new or progressive infiltrate on chest X-ray, leucocytosis \& purulent trachea-bronchial secretions. Aggressive surveillance is vital in understanding local factors leading to VAP and the microbiologic milieu of a given unit in the hospital. Judicious antibiotic usage is essential, as resistant micro organisms are known to plague intensive care units and critically ill patients. After diagnosing VAP, the clinician should promptly institute the appropriate antibiotic regimen, defined by sensitivity of the organism cultured. Dosing the drug is of paramount importance, since 
the first 48 hours is critical for patient survival. Starting high end antibiotics for ventilated patients to treat colonizers, leads to proliferation of MDR (multi-drug resistant) pathogens.

The organisms recovered have an impact on the clinical prognosis, with higher mortality rates seen in VAP caused by Pseudomonas aeruginosa and Acinetobacter spp. Numerous studies have showed that prior \& concurrent antibiotic therapy decreases the sensitivity and negative predictive value of Gram staining, quantitative and semiquantitative cultures. To potentially improve the accuracy of the diagnosis of VAP and avoiding unnecessary antibiotic use \& abuse, various studies have investigated the role of quantitative cultures of respiratory secretions. Bronchoscopic samples are processed by quantitative cultures and the threshold values have been derived, \& expressed in CFU (colony forming units) per milliliter ${ }^{1}$.

If the number of $\mathrm{CFU} / \mathrm{ml}$ is equal to or exceeds the threshold values for the particular technique, a diagnosis of pneumonia is made. Threshold values often employed for diagnosing pneumonia by quantitative cultures are $\geq 10^{5}$ to $10^{6}$ and $\geq$ $10^{4}$ for QEA and bronchoscopic BAL respectively, with $\geq 10^{5} \mathrm{CFU} / \mathrm{ml}$ being the most widely accepted value for QEA $^{1}$. These cut-off values for diagnosing VAP are based in part on the findings of quantitative cultures obtained from infected lung tissue, and the volume \& dilution of the respiratory secretions retrieved by the technique. For instance, BAL collects approximately $1 \mathrm{ml}$ of secretions in 10-100 ml of effluent. This corresponds to a dilution factor of $1 / 10$ to 1/100.Several investigators have confirmed that with pneumonia, pathogens are present in lower respiratory tract inflammatory secretions at concentrations of at least $10^{5}$ to
$10^{6} \mathrm{CFU} / \mathrm{ml}$; contaminants are generally present at less than $10^{4} \mathrm{CFU} / \mathrm{ml}$.

\section{Need for the Study}

Ventilator-associated pneumonia (VAP) increases the crude mortality rate by $2-10$ times, and the hospital costs by increasing the length of hospital stay and the need for more expensive antibiotics. This study is designed to assess the role of quantitative endotracheal aspirate (QEA) in the diagnosis of VAP in a tertiary care hospital in South India.

The main objective of this study includes, to assess the role of quantitative culture of endotracheal aspirates in correlating with the diagnosis of VAP in ICU patients.

\section{Inclusion Criteria}

All patients admitted in ICU who were on mechanical ventilation for more than 48 hours.

\section{Exclusion Criteria}

Patients admitted in ICU who were not on mechanical ventilation

\section{Methodology}

Identification of patients with a clinical diagnosis of Ventilator-associated pneumonia (VAP) using CPIS (clinical pulmonary infection score)

Culture \& sensitivity of organisms obtained from endotracheal aspirate

Quantitation of culture aspirate

Corroboration of clinical diagnosis of VAP with quantitative culture of endotracheal aspirate $\geq 10^{5} \mathrm{CFU} / \mathrm{ml}$ 


\section{Study Design}

Prospective, observational study

\section{Materials and Methods}

About 542 ICU patients who were on mechanical ventilation for more than 48 hours were screened for clinical signs of VAP, out of which 22 patients were diagnosed with Ventilator-associated pneumonia (VAP). Endotracheal aspirate culture \& sensitivity of those 22 patients were analysed.

\section{Results and Discussion}

About 542 ICU patients on mechanical ventilation were included in the study. Those patients who were on ventilator support for more than 48 hours were screened for signs of Ventilator-associated pneumonia (VAP), using CPIS (clinical pulmonary infection score) denoted by rise in temperature, white blood cell count > $10,000 /$ cu.mm, total number of ventilator days, development of new infiltrate shadows on chest X-ray and presence of purulent trachea- bronchial secretions. 22 patients were diagnosed with VAP based on those criteria. Quantitative culture of endotracheal aspirates in all 22 mechanically ventilated patients diagnosed with VAP were $\geq 10^{5}$ $\mathrm{CFU} / \mathrm{ml}$.

Total number of ventilator days: 3056

Number of patients with VAP: 22

VAP rate: 7.1 per 1000 ventilator days

Diagnosing VAP requires a high clinical suspicion combined with bed-side examination, radiography and microbiological analysis of respiratory secretions. Initially CPIS (clinical pulmonary infection score) combined with quantitative cultures can be used for baseline evaluation of the clinical status. Antibiotic administration should be promptly initiated when VAP is suspected, with a broad coverage of organisms. Knowledge of local antibiograms should guide the choice of antibiotics. For patients already on antibiotics at the time of suspected VAP, the clinician must choose antibiotics from different classes, as it is likely that resistance to "in-use" antibiotics has developed. On the third day, assessment of quantitative culture results and sensitivity at this juncture is prudent, as it may permit early antibiotic deescalation.

\section{Pathogens Isolated from Culture of Endotracheal Aspirates}

\begin{tabular}{|l|l|}
\hline Pseudomonas aeruginosa & $4 \mathrm{pts}$ \\
\hline $\begin{array}{l}\text { Klebsiella pneumoniae \& } \\
\text { Pseudomonas aeruginosa }\end{array}$ & 2 \\
\hline Escherichia coli ESBL) & 2 \\
\hline $\begin{array}{l}\text { Acinetobacter baumanii \& } \\
\text { Klebsiella pneumoniae }\end{array}$ & 2 \\
\hline Klebsiella pneumoniae - ESBL & 2 \\
\hline Acinetobacter baumanii & 4 \\
\hline $\begin{array}{l}\text { Acinetobacter baumanii } \\
\text { (carbapenemase producer) }\end{array}$ & 2 \\
\hline $\begin{array}{l}\text { Acinetobacter baumanii \& } \\
\text { Pseudomonas aeruginosa }\end{array}$ & 2 \\
\hline Klebsiella oxytoca & 1 \\
\hline $\begin{array}{l}\text { Escherichia coli }(\text { ESBL) \& } \\
\text { Pseudomonas aeruginosa }\end{array}$ & 1 \\
\hline
\end{tabular}

Most studies have concluded that the sensitivities of non-bronchoscopic and bronchoscopic quantitative techniques are comparable. However, the overall concordance in some studies has been only approximately $80 \%$. Superior specificity of quantitative compared to non-quantitative and semi- quantitative culture techniques permits us to more confidently de-escalate antibiotics, thereby eliminating bacterial resistance. In addition, a negative quantitative culture compels us to more 
aggressively search for other non-infectious and non-pulmonary causes of the patient's clinical presentation.

Numerous factors can influence the results of QEA cultures, including the time of pneumonia onset, skill of the person collecting the sample, adequacy of specimen, technical aspects such as processing or delay in sample transport to the lab, special microbial populations in patients with COPD who may have relatively high bacterial counts without pneumonia, and prior \& concurrent antibiotic therapy.

Due to these potential limitations, it is important to bear in mind that a quantitative culture that exceeds the threshold value is not diagnostic of VAP by itself. Falsepositive QEA cultures could be secondary to bronchiolitis, colonization or oro-pharyngeal contamination. Likewise a result below these threshold values does not rule out the presence of pneumonia, particularly if prior antibiotic therapy has been instituted. While higher bacterial counts correlate with a higher likelihood of VAP, lower counts are associated with a lower probability.

In a study done by Chien Liang Wu et.al, data regarding quantitative culture of endotracheal aspirates correlating with protected specimen brushings and bronchoalveolar lavage in patients with treatment failure diagnosed with VAP have been analysed. QEA is non-invasive, and early investigation by QEA would be helpful to the clinician in decision-making with regard to antibiotic use.

In conclusion, this study does confirm the microbiological corroboration in predicting the diagnosis of VAP, which is re-inforced by the role of quantitative culture of endotracheal aspirates in ICU patients on mechanical ventilation for more than 48 hours. Aggressive surveillance is vital in understanding local factors leading to VAP and the microbiologic milieu.

\section{References}

Baker, A.M., Bowton, D.L., Haponik, E.F. 1995. CHEST, 107: 85-95.

Chen Liang Wu, et al. 2002. CHEST, 122: 662-668.

Chiranjay Mukhopadhyay, et al. 2010. Int. J. Infect. Control, 6.

Jourdain, B., Novara, A., Joly-Guillou, M.L., Dombret, M.C., et al. 1995. Am. J. Respir. Crit. Care Med., 152: 241-246.

Marquette, C.H., et al. 1995. Am. J. Respir. Crit. Care Med., 151: 1878-1888

Mayhall, et al. Infect. Dis. N. Am., Vol. 11: 427-457.

Steven, M., Koenig, Jonathan, D., Truwit. 2006. Clin. Microbiol. Rev., Vol. 19, No. 4: pp. 637-657.

\section{How to cite this article:}

Jayashree, V., Karthika Jayakumar and Parvathi, S. 2016. Microbiological Corroboration for Diagnosis of VAP (Ventilator Associated Pneumonia) by Quantitative Culture of Endotracheal Aspirate (QEA) in ICU Patients in a Tertiary Hospital, South India. Int.J.Curr.Microbiol. App.Sci.5(4): 371-374. doi: http://dx.doi.org/10.20546/ijcmas.2016.504.044 